\title{
Low serum adiponectin level is associated with metabolic syndrome and is an independent marker of peripheral arterial stiffness in hypertensive patients
}

\author{
Ming-Chun Chen' ${ }^{1}$, Chung-Jen Lee ${ }^{2}$, Chiu-Fen Yang ${ }^{3}$, Yu-Chih Chen ${ }^{3}$, Ji-Hung Wang ${ }^{3,4^{*}+}$ \\ and Bang-Gee Hsu ${ }^{4,5^{*}}$ (1)
}

\begin{abstract}
Background: Adiponectin has been implicated in metabolic syndrome (MetS) and arterial stiffness (AS). We aim to determine the relationship between serum adiponectin concentration as well as peripheral AS in hypertensive patients.

Methods: Fasting blood samples were obtained from 101 hypertensive patients. Brachial-ankle pulse wave velocity (baPWV) was measured with an automatic pulse wave analyzer. Serum adiponectin concentrations were determined by using an enzyme immunoassay kit. A baPWV $>14.0 \mathrm{~m} / \mathrm{s}$ was defined as high AS.

Results: MetS and high AS were present in 62.4 and $71.3 \%$ of the study population. Adiponectin was inversely associated with MetS and high AS (both $P<0.001)$. Serum higher high-density lipoprotein cholesterol (HDL-C) $(P=0.012)$, triglycerides $(P=0.001)$, C-reactive protein $(P<0.001)$, insulin $(P=0.027)$, body weight $(P=0.002)$, waist circumference (WC, $P<0.001)$, body mass index $(P=0.001)$ bilateral-baPWV $(P<0.001)$, systolic blood pressure $(S B P, P<0.001)$, diastolic blood pressure (DBP, $P=0.012)$, pulse pressure $(P=0.019)$, homeostasis model assessment of insulin resistance (HOMA1-IR $(P=0.026)$ and HOMA2-IR $(P=0.020)$ ) and lower glomerular filtration rate $(G F R, P=0.029)$ were significantly associated with high AS. Multivariate logistic regression analysis of the factors significantly associated with AS revealed that adiponectin [odds ratio: $0.932,95 \%$ confidence interval $(C I) 0.881-0.985, P=0.012$ ], and SBP (odds ratio: 1.059, 95\% Cl 1.008-1.113, $P=0.022$ ) were the independent predictors of arterial stiffness in hypertensive patients. Subgroup analysis revealed that SBP (odds ratio: 1.126, 95\% Cl 1.024-1.237, $P=0.014$ ) and GFR (odds ratio: $0.858,95 \% \mathrm{Cl} 0.739-0.996, P=0.043$ ) were the independent predictors of arterial stiffness in hypertensive patients without MetS; adiponectin (odds ratio: $0.909,95 \% \mathrm{Cl} 0.931-0.996, P=0.040$ ) was the independent predictor of arterial stiffness in hypertensive patients with MetS.
\end{abstract}

Conclusions: Hypoadiponectinemia has positive association with MetS and peripheral AS in hypertensive patients. Keywords: Adiponectin, Peripheral arterial stiffness, Metabolic syndrome, Hypertension

\footnotetext{
*Correspondence: abanggeelily@gmail.com; gee.lily@msa.hinet.net ${ }^{\dagger} \mathrm{Ji}$-Hung Wang and Bang-Gee Hsu contributed equally to this study ${ }^{3}$ Division of Cardiology, Buddhist Tzu Chi General Hospital, No. 707, Section 3, Chung-Yang Road, Hualien 97002, Taiwan

${ }^{5}$ Division of Nephrology, Buddhist Tzu Chi General Hospital, No. 707, Section 3, Chung-Yang Road, Hualien 97002, Taiwan

Full list of author information is available at the end of the article
} 


\section{Background}

Metabolic syndrome (MetS), a cluster of interrelated cardiometabolic risk factors, including visceral obesity, hypertension, hyperglycemia, elevated triglycerides (TG), and decreased high-density lipoprotein cholesterol (HDL-C) levels, is an independent risk factor for type 2 diabetes mellitus (DM) and cardiovascular (CV) disease [1]. The diabetes and heart disease risks are expected to rise by 2- to 5-fold over the next 5-10 years in patients with MetS compared with individuals without MetS [2].

Adiponectin secreted by adipocytes, contains a 247amino acid protein with four differentiable domains [3]. Adiponectin plays an important role in obesity, insulin resistance (IR), MetS, and CV disease through its antiinflammatory, anti-diabetic, and anti-atherogenic properties $[4,5]$.

Arterial stiffness, a pathological condition accompanying vascular damage, is one of the characteristics of $\mathrm{CV}$ disease. Pulse wave velocity (PWV), one of the widely used noninvasive methods to evaluate arterial stiffness, reflects segmental arterial elasticity in clinical practice [6]. Brachial-ankle pulse wave velocity (baPWV), which is more easily applied than carotid-femoral PWV, has been used as a surrogate of peripheral arterial stiffness and a marker for screening $\mathrm{CV}$ risk in the general population, in diabetes, and in hypertensive patients [6-9]. One meta-analysis revealed increased pooled relative risks for total CV events, CV mortality, and all-cause mortality for patients with high versus low baPWV [10].

MetS is associated with a higher prevalence of $\mathrm{CV}$ events and arterial stiffness [8]. A low serum adiponectin level is associated with both central elastic and peripheral muscular arterial stiffness in non-treated hypertensive patients $[11,12]$. However, in the population of MetS patients, it remains uncertain whether decreased serum adiponectin is an independent risk factor for arterial stiffness. Thus, the present study provides further evidence of the relationship between decreased serum adiponectin and MetS. In addition, we investigated whether low serum adiponectin is independently associated with peripheral arterial stiffness evaluated by baPWV.

\section{Methods}

\section{Patients}

A total of 101 hypertensive participants were enrolled in this cross-sectional study conducted between January and December 2012 in the cardiovascular outpatient department at Buddhist Tzu Chi General Hospital, Hualien, Taiwan and the use of anti-hypertensive drugs for control blood pressure (BP) were according the Eighth Joint National Committee (JNC 8) guideline. By using standard mercury sphygmomanometers with appropriate cuff sizes, the BP of all patients was measured on the right arm by trained staff after sitting for at least $10 \mathrm{~min}$ in the morning. Systolic BP (SBP) and diastolic BP (DBP) were measured three times at 5-min intervals and were averaged for analysis. Hypertension was defined as $\mathrm{SBP} \geq 140 \mathrm{mmHg}$, and/or DBP $\geq 90 \mathrm{mmHg}$, or having received any anti-hypertensive medication in the past 2 weeks among patients enrolled in this study. The Protection of Human Subjects Institutional Review Board of Tzu-Chi University and Hospital approved the study. All patients provided their informed consent before participation. Exclusion criteria included acute infection, acute myocardial infarction, and pulmonary edema at the time of blood sampling, or refusal to provide informed consent for the study.

\section{Anthropometric analysis}

Body weight (BW) and height were measured with the participants in light clothing without shoes to the nearest $0.5 \mathrm{~kg}$ and half centimeter, respectively. Waist circumference (WC) was measured at the midpoint between the lowest ribs and the iliac crest with the hands on the hips. Body mass index (BMI) was calculated using the Quetelet's formula as the weight in kilograms divided by the height in meters squared $[2,6,13,14]$.

\section{Biochemical investigations}

After 8-12 h overnight fasting, blood samples (approximately $5 \mathrm{~mL}$ ) of all participants were immediately centrifuged at $3000 \mathrm{~g}$ for $10 \mathrm{~min}$. Serum levels of blood urea nitrogen (BUN), creatinine (Cre), fasting glucose, total cholesterol (TCH), TG, HDL-C, low-density lipoprotein cholesterol (LDL-C), total calcium, phosphorus, and $\mathrm{C}$-reactive protein (CRP) were determined by using an autoanalyzer (COBAS Integra 800, Roche Diagnostics, Basel, Switzerland) $[2,6,13,14]$. Serum adiponectin (SPI-BIO, Montigny le Bretonneux, France) and serum intact parathyroid hormone (iPTH) (Diagnostic Systems Laboratories, Texas, USA) concentrations were measured using a commercially available enzyme immunoassay and enzyme-linked immunosorbent assays, respectively [13]. The Modification of Diet in Renal Disease (MDRD) equation was used in this study for the calculation of estimated glomerular filtration rate (GFR).

\section{Metabolic syndrome and its components}

The prevalence of MetS was determined by using the international diabetes federation definition [15], which includes central obesity with a waist circumference $\geq 90 \mathrm{~cm}$ (men) or $\geq 80 \mathrm{~cm}$ (women) (Chinese criteria) and meeting two or more of the following criteria: fasting serum glucose of $\geq 100 \mathrm{mg} / \mathrm{dL}$, TG of $\geq 150 \mathrm{mg} /$ $\mathrm{dL}, \mathrm{HDL}-\mathrm{C}$ level $<40 \mathrm{mg} / \mathrm{dL}$ in $\mathrm{men}$ or $<50 \mathrm{mg} / \mathrm{dL}$ in women or BP of $\geq 130 / 85 \mathrm{mmHg}$. Anti-hypertensive 
medication usage was considered as indicative of high $\mathrm{BP}$ in this analysis. Type $2 \mathrm{DM}$ was defined using the World Health Organization criteria [16]. Individuals were classified as DM if the fasting plasma glucose was either $126 \mathrm{mg} / \mathrm{dL}$ or more or if the 2-h glucose during an oral glucose tolerance test was $\geq 200 \mathrm{mg} / \mathrm{dL}$ or if he/she was using diabetes medication (oral or insulin). Serum insulin levels were measured using the microparticle enzyme immunosorbent assay method by an autoanalyzer (Abbott Laboratories, Abbott Park, IL, USA). Insulin resistance was evaluated using a homeostasis model assessment of insulin resistance (HOMAIR) as follows: HOMA1-IR = fasting plasma glucose $(\mathrm{mg} / \mathrm{dL}) \times$ fasting serum insulin $(\mu \mathrm{U} / \mathrm{mL}) / 405[2,17]$; HOMA2-IR, is computer model which better reflects human physiology and is recalibrated to modern insulin assays (The HOMA2-IR model is available from http:// www.OCDEM.ox.ac.uk) [18].

\section{Brachial-ankle pulse wave velocity measurements}

BaPWV measurements were arranged immediately on the same day as blood sampling. After a 10-min rest in a quiet, temperature-controlled room, all participants were subjected to measurements in a supine position per the recommendations for user procedures in clinical applications of arterial stiffness. Heart rate and blood pressure (mean of three readings) were checked with an automatic upper-arm oscillometric device. The baPWV was measured in the right or left brachial artery to the ankle segments using an automatic pulse wave analyzer (VaSera VS-1000, Fukuda Denshi Co. Ltd., Tokyo, Japan) $[6,14]$. In brief, cuffs were applied to the four extremities and electrocardiographic electrodes were attached to the upper arm. A microphone was placed on the sternal angle for phonocardiography. The subjects then rested in a supine position for $5 \mathrm{~min}$. The baPWV was calculated by dividing the distance from the aortic valve to the ankle artery by the sum of the difference between the time the pulse waves were transmitted to the brachium and the time the same waves were transmitted to the ankle, and the time difference between the second heart sound on the phonocardiogram and the notch of the brachial pulse wave. To minimize cuff inflation effects on blood flow dynamics, pulse waves were measured with the cuffs inflated to less than the DBP $(50 \mathrm{mmHg})$. In this study, left or right baPWV values of $>14.0 \mathrm{~m} / \mathrm{s}$ were used to define the high arterial stiffness group [6].

\section{Statistical analysis}

All statistical analyzes were performed using the statistical package for the social sciences (SPSS) version 19.0 (SPSS Inc., Chicago, IL, USA). The distribution pattern of the variables was checked using the
Kolmogorov-Smirnov test. Normally distributed variables are expressed as mean \pm standard deviation and comparisons between patients were performed using the Student's independent $t$ test (two-tailed). Data not normally distributed are expressed as medians and interquartile ranges, and comparisons between patients were performed by using the Mann-Whitney U test (TG, fasting glucose, CRP, iPTH, insulin, HOMA1-IR, HOMA2IR, and adiponectin). Data expressed as the number of patients were analyzed by the $\chi^{2}$ test. Because TG, fasting glucose, CRP, iPTH, insulin, HOMA1-IR, HOMA2IR, and adiponectin were not normally distributed, they underwent base 10 logarithmic transformations to achieve normality. Variables that were significantly associated with arterial stiffness in hypertensive patients or hypertensive patients without MS or hypertensive patients with MS were tested for independence by multivariate logistic regression analysis. A $P$ value $<0.05$ was considered statistically significant.

\section{Results}

Demographic, clinical, and biochemical characteristics of the 101 hypertensive patients with or without MetS are presented in Table 1. A total of 44 patients $(43.6 \%)$ had DM and 67 patients $(66.3 \%)$ had a medical history of CAD. Sixty-three patients (62.4\%) with MetS had lower serum adiponectin $(P<0.001)$ and HDL-C $(P=0.002)$ levels, higher serum TG $(P<0.001)$, fasting glucose $(P<0.001)$, CRP $(P<0.001)$, insulin $(P=0.004)$ levels, elevated BW $(P<0.001)$, WC $(P<0.001)$, BMI $(P<0.001)$, left and right-baPWV $(P<0.001)$, SBP $(P=0.014)$, HOMA1-IR $(P=0.001)$, HOMA2-IR $(P=0.002)$ values, and higher percentages of type $2 \mathrm{DM}(P=0.039)$ than those in the non-MetS group.

The demographics, clinical characteristics, biochemical data, and comorbidities of our 101 hypertensive patients with or without peripheral arterial stiffness are presented in Table 2. Seventy-two patients (71.3\%) with high arterial stiffness had lower serum adiponectin $(P<0.001)$ and HDL-C $(P=0.012)$ levels, higher serum TG $(P=0.001)$, CRP $(P<0.001)$, insulin $(P=0.027)$ levels, elevated BW $(P=0.002)$, WC $(P<0.001)$, BMI $(P=0.001)$, left and right-baPWV $(P<0.001)$, SBP $(P<0.001)$, DBP $(P=0.012)$, pulse pressure $(P=0.019)$, HOMA1-IR $(P=0.026)$, HOMA2-IR $(P=0.020)$ values and lower GFR $(P=0.029)$ than those in the low arterial stiffness group. Uni- and multivariate linear analyses of the clinical variables associated with left and right baPWV levels in hypertensive patients are shown in Additional file 1: Table S1 and Table S2.

The drugs used included angiotensin-converting enzyme inhibitors (ACEi; $\mathrm{n}=37 ; 36.6 \%$ ), angiotensin receptor blockers (ARB; $\mathrm{n}=56 ; 55.4 \%$ ), $\beta$-blockers 
Table 1 Clinical variables of the 101 hypertensive patients with or without metabolic syndrome

\begin{tabular}{|c|c|c|c|c|}
\hline Variables & All participants $(n=101)$ & $\begin{array}{l}\text { No metabolic syndrome } \\
(\mathrm{n}=38)\end{array}$ & $\begin{array}{l}\text { Metabolic syndrome } \\
(n=63)\end{array}$ & $P$ value \\
\hline Age (years) & $64.88 \pm 9.58$ & $66.13 \pm 10.14$ & $64.13 \pm 9.23$ & 0.311 \\
\hline Height (cm) & $161.27 \pm 8.36$ & $162.22 \pm 7.24$ & $160.70 \pm 8.98$ & 0.377 \\
\hline Body weight (kg) & $69.73 \pm 13.01$ & $63.34 \pm 10.80$ & $73.59 \pm 12.78$ & $<0.001^{*}$ \\
\hline Waist circumference (cm) & $93.37 \pm 11.55$ & $84.82 \pm 9.44$ & $98.52 \pm 9.50$ & $<0.001^{*}$ \\
\hline Body mass index $\left(\mathrm{kg} / \mathrm{m}^{2}\right)$ & $26.71 \pm 3.87$ & $23.99 \pm 3.24$ & $28.34 \pm 3.27$ & $<0.001^{*}$ \\
\hline Left baPWV (m/s) & $15.22 \pm 3.45$ & $12.65 \pm 3.06$ & $16.77 \pm 2.67$ & $<0.001^{*}$ \\
\hline Right baPWV (m/s) & $15.32 \pm 3.69$ & $12.46 \pm 3.01$ & $17.04 \pm 2.91$ & $<0.001^{*}$ \\
\hline Systolic blood pressure $(\mathrm{mmHg})$ & $133.44 \pm 16.68$ & $128.21 \pm 13.70$ & $136.59 \pm 17.60$ & $0.014^{*}$ \\
\hline Diastolic blood pressure $(\mathrm{mmHg})$ & $74.52 \pm 10.73$ & $72.34 \pm 10.64$ & $75.84 \pm 10.65$ & 0.113 \\
\hline Pulse pressure $(\mathrm{mmHg})$ & $58.91 \pm 14.92$ & $55.87 \pm 12.71$ & $60.75 \pm 15.92$ & 0.112 \\
\hline Total cholesterol (mg/dL) & $174.10 \pm 40.81$ & $176.95 \pm 39.80$ & $172.38 \pm 41.62$ & 0.588 \\
\hline Triglycerides (mg/dL) & $127.00(91.00-177.00)$ & $107.00(73.75-128.50)$ & $150.00(104.00-218.00)$ & $<0.001^{*}$ \\
\hline $\mathrm{HDL}-\mathrm{C}(\mathrm{mg} / \mathrm{dL})$ & $45.06 \pm 13.07$ & $50.08 \pm 14.25$ & $42.03 \pm 11.39$ & $0.002^{*}$ \\
\hline LDL-C (mg/dL) & $102.29 \pm 31.55$ & $105.16 \pm 31.71$ & $100.56 \pm 31.58$ & 0.480 \\
\hline Fasting glucose (mg/dL) & $108.00(96.00-145.50)$ & $97.50(89.75-115.25)$ & $122.00(103.00-161.00)$ & $<0.001^{*}$ \\
\hline Blood urea nitrogen (mg/dL) & $17.38 \pm 5.73$ & $16.13 \pm 3.97$ & $18.13 \pm 6.48$ & 0.090 \\
\hline Creatinine $(\mathrm{mg} / \mathrm{dL})$ & $1.13 \pm 0.32$ & $1.09 \pm 0.28$ & $1.15 \pm 0.35$ & 0.318 \\
\hline Glomerular filtration rate (mL/min) & $68.93 \pm 19.98$ & $72.82 \pm 19.55$ & $66.59 \pm 20.02$ & 0.130 \\
\hline Total calcium (mg/dL) & $9.14 \pm 0.38$ & $9.03 \pm 0.38$ & $9.20 \pm 0.36$ & $0.035^{*}$ \\
\hline Phosphorus (mg/dL) & $3.51 \pm 0.52$ & $3.40 \pm 0.46$ & $3.58 \pm 0.55$ & 0.095 \\
\hline Calcium-phosphorous product $\left(\mathrm{mg}^{2} / \mathrm{dL}^{2}\right)$ & $32.09 \pm 5.25$ & $30.71 \pm 4.66$ & $32.92 \pm 5.45$ & $0.041^{*}$ \\
\hline Intact parathyroid hormone (pg/mL) & $46.35(32.40-61.90)$ & $49.60(34.25-67.58)$ & $44.15(28.88-57.55)$ & $0.009^{*}$ \\
\hline C-reactive protein (mg/dL) & $0.20(0.14-0.27)$ & $0.16(0.12-0.19)$ & $0.24(0.17-0.36)$ & $<0.001^{*}$ \\
\hline Insulin (ulU/mL) & $11.99(7.98-25.16)$ & $9.86(6.06-15.14)$ & $15.22(9.22-28.99)$ & $0.004^{*}$ \\
\hline HOMA1-IR & $3.57(2.27-7.45)$ & $2.37(1.50-4.58)$ & $4.35(2.93-8.72)$ & $0.001^{*}$ \\
\hline HOMA2-IR & $1.63(1.10-3.30)$ & $1.32(0.81-1.97)$ & $2.14(1.33-3.91)$ & $0.002^{*}$ \\
\hline Adiponectin $(\mu \mathrm{g} / \mathrm{mL})$ & $28.40(23.09-39.90)$ & 36.92 (26.53-47.17) & $25.55(21.00-33.66)$ & $<0.001^{*}$ \\
\hline Female (\%) & $34(33.7)$ & $9(23.7)$ & $25(39.7)$ & 0.099 \\
\hline Diabetes (\%) & $44(43.6)$ & $9(23.7)$ & $35(55.6)$ & $0.002^{*}$ \\
\hline
\end{tabular}

Values for continuous variables given as mean \pm standard deviation and compared by Student's $t$ test; variables not normally distributed given as medians and interquartile range and compared by Mann-Whitney $\mathrm{U}$ test; values are presented as number (\%), and analysis was performed using the Chi square test

baPWV brachial-ankle pulse wave velocity, HDL-C high-density lipoprotein cholesterol, LDL-C low-density lipoprotein cholesterol, HOMA-IR homeostasis model assessment of insulin resistance

${ }^{*} P<0.05$ was considered statistically significant

( $\mathrm{n}=56 ; 55.4 \%)$, calcium channel blockers $(\mathrm{CCB} ; \mathrm{n}=44$; $43.6 \%)$, thiazides $(n=11 ; 10.9 \%)$, statins $(n=52 ; 51.2 \%)$, and fibrates $(\mathrm{n}=11 ; 10.9 \%)$. Peripheral arterial stiffness did not differ statistically by sex, CAD, or use of ACEi, $\mathrm{ARB}, \beta$-blockers, $\mathrm{CCB}$, thiazides, statins, or fibrates, but there were statistically significant differences in diabetes and MetS (both $P<0.001$ ) among hypertensive patients (Table 3).

Subgroup analysis of arterial stiffness in hypertensive patients with or without MetS is presented in Table 4. High arterial stiffness patients without MetS had advanced age $(P=0.023)$, higher bilateral baPWV (both $P<0.001)$, and SBP $(P<0.001)$ values, and lower GFR $(P=0.016)$ than those in the low arterial stiffness group.
Hypertensive patients with MetS and baPWV values $>14 \mathrm{~m} / \mathrm{sec}$ had higher triglyceride $(P<0.026)$ and CRP $(P=0.015)$ levels, and lower HDL-C $(P=0.003)$ and adiponectin $(P<0.001)$ levels than hypertensive patients with MetS with baPWV values $\leq 14 \mathrm{~m} / \mathrm{sec}$.

Multivariate logistic regression analysis of the factors (diabetes, age, BW, WC, BMI, SBP, DBP, pulse pressure, triglyceride, HDL-C, GFR, CRP, insulin, HOMA1-IR, HOMA2-IR, and adiponectin) significantly associated with arterial stiffness revealed that adiponectin (odds ratio: 0.932 , 95\% confidence interval (CI) 0.881-0.985, $P=0.012$ ), and SBP (odds ratio: 1.059 , 95\% CI 1.008$1.113, P=0.022)$ were the independent predictors of arterial stiffness in hypertensive patients. Subgroup 
Table 2 Clinical variables of the 101 hypertensive patients with or without arterial stiffness

\begin{tabular}{|c|c|c|c|}
\hline Variables & Low arterial stiffness group $(n=29)$ & High arterial stiffness group $(n=72)$ & $P$ value \\
\hline Age (years) & $64.00 \pm 8.90$ & $65.24 \pm 9.88$ & 0.560 \\
\hline Height (cm) & $160.21 \pm 6.80$ & $161.70 \pm 8.93$ & 0.419 \\
\hline Body weight (kg) & $63.59 \pm 10.28$ & $72.21 \pm 13.23$ & $0.002^{*}$ \\
\hline Waist circumference (cm) & $86.03 \pm 10.36$ & $96.32 \pm 10.72$ & $<0.001^{*}$ \\
\hline Body mass index $\left(\mathrm{kg} / \mathrm{m}^{2}\right)$ & $24.80 \pm 3.81$ & $27.48 \pm 3.65$ & $0.001^{*}$ \\
\hline Left baPWV (m/s) & $11.24 \pm 2.40$ & $16.82 \pm 2.32$ & $<0.001^{*}$ \\
\hline Right baPWV (m/s) & $11.15 \pm 2.48$ & $16.99 \pm 2.60$ & $<0.001^{*}$ \\
\hline Systolic blood pressure (mmHg) & $123.79 \pm 14.84$ & $137.32 \pm 15.87$ & $<0.001^{*}$ \\
\hline Diastolic blood pressure $(\mathrm{mmHg})$ & $70.34 \pm 9.08$ & $76.21 \pm 10.93$ & $0.012^{*}$ \\
\hline Pulse pressure $(\mathrm{mmHg})$ & $53.45 \pm 13.83$ & $61.11 \pm 14.86$ & $0.019^{*}$ \\
\hline Total cholesterol (mg/dL) & $178.93 \pm 40.20$ & $172.15 \pm 41.17$ & 0.453 \\
\hline Triglycerides (mg/dL) & $105.00(63.50-130.00)$ & $143.00(101.75-208.50)$ & $0.001^{*}$ \\
\hline $\mathrm{HDL}-\mathrm{C}(\mathrm{mg} / \mathrm{dL})$ & $50.17 \pm 11.71$ & $43.00 \pm 13.10$ & $0.012^{*}$ \\
\hline $\mathrm{LDL}-\mathrm{C}(\mathrm{mg} / \mathrm{dL})$ & $106.62 \pm 30.51$ & $100.54 \pm 32.00$ & 0.384 \\
\hline Fasting glucose (mg/dL) & $99.00(93.00-123.50)$ & $113.00(98.00-158.50)$ & 0.066 \\
\hline Blood urea nitrogen (mg/dL) & $16.72 \pm 5.94$ & $17.64 \pm 5.67$ & 0.471 \\
\hline Creatinine (mg/dL) & $1.05 \pm 0.33$ & $1.16 \pm 0.32$ & 0.132 \\
\hline Glomerular filtration rate (mL/min) & $75.76 \pm 20.76$ & $66.18 \pm 19.11$ & $0.029^{*}$ \\
\hline Total calcium (mg/dL) & $9.07 \pm 0.36$ & $9.16 \pm 0.39$ & 0.286 \\
\hline Phosphorus (mg/dL) & $3.46 \pm 0.51$ & $3.53 \pm 0.53$ & 0.531 \\
\hline Calcium-phosphorous product $\left(\mathrm{mg}^{2} / \mathrm{dL}^{2}\right)$ & $31.40 \pm 5.05$ & $32.37 \pm 5.34$ & 0.405 \\
\hline Intact parathyroid hormone (pg/mL) & $51.10(36.15-69.05)$ & $44.40(29.00-58.60)$ & 0.224 \\
\hline C-reactive protein (mg/dL) & $0.16(0.12-0.20)$ & $0.23(0.16-0.32)$ & $<0.001^{*}$ \\
\hline Insulin (ulU/mL) & $9.61(5.52-18.66)$ & $13.79(9.10-27.04)$ & $0.027^{*}$ \\
\hline HOMA1-IR & $2.76(1.62-4.70)$ & $3.89(2.59-8.55)$ & $0.026^{*}$ \\
\hline HOMA2-IR & $1.30(0.78-2.38)$ & $1.96(1.27-3.70)$ & $0.020^{*}$ \\
\hline Adiponectin ( $\mu \mathrm{g} / \mathrm{mL})$ & $39.91(27.06-52.88)$ & $26.89(21.15-34.41)$ & $<0.001^{*}$ \\
\hline
\end{tabular}

Values for continuous variables given as mean \pm standard deviation and compared by Student's $t$ test; variables not normally distributed given as medians and interquartile range and compared by Mann-Whitney $\mathrm{U}$ test

baPWV brachial-ankle pulse wave velocity, HDL-C high-density lipoprotein cholesterol, LDL-C low-density lipoprotein cholesterol, iPTH intact parathyroid hormone, HOMA-IR homeostasis model assessment of insulin resistance

* $P<0.05$ was considered statistically significant

analysis revealed that SBP (odds ratio: 1.126, 95\% CI 1.024-1.237, $P=0.014$ ) and GFR (odds ratio: $0.858,95 \%$ CI $0.739-0.996, P=0.043$ ) were the independent predictors of arterial stiffness in hypertensive patients without MetS, while adiponectin (odds ratio: 0.909, 95\% CI 0.931-0.996, $P=0.040$ ) was the independent predictor of arterial stiffness in hypertensive patients with MetS (Table 5).

\section{Discussion}

Serum adiponectin levels were significantly lower in hypertensive patients with MetS and had association with arterial stiffness in hypertensive patients. Decreased serum adiponectin level is associated with the development of arterial stiffness in hypertensive individuals with MetS, but this association lost significance in the group without MetS.
Insulin resistance with compensatory hyperinsulinemia plays a central role in MetS [2]. Insulin metabolism is interrelated with adiponectin secretion and action [19]. In recent years, adiponectin has been recognized as an insulin sensitizer involved in energy metabolism and regulation of many biological processes, such as migration, proliferation, apoptosis, and inflammation [20]. Epidemiological studies found that decreased serum total and high-molecular-weight adiponectin were associated with MetS in 546 Japanese-Americans and in 750 Japanese during a 3-4-year follow up [21, 22]. Another study with 2044 participants showed that hypoadiponectinemia was significantly associated with incident MetS, even after adjusting for BMI, CRP, and HOMA-IR [23]. Recently, Lindberg et al. even reported that hypoadiponectinemia at baseline and during a 9-year follow up had significant positive correlations with incident MetS independently of 
Table 3 Baseline characteristics of the 101 hypertensive patients with or without arterial stiffness

\begin{tabular}{|c|c|c|c|}
\hline Characteristic & Low arterial stiffness group (\%) & High arterial stiffness group (\%) & $P$ value \\
\hline \multicolumn{4}{|l|}{ Gender } \\
\hline Male & $19(65.5)$ & $48(66.7)$ & \multirow[t]{2}{*}{0.912} \\
\hline Female & $10(34.5)$ & $24(33.3)$ & \\
\hline \multicolumn{4}{|l|}{ Diabetes } \\
\hline No & $21(72.4)$ & $36(50.0)$ & \multirow[t]{2}{*}{$<0.001^{*}$} \\
\hline Yes & $8(27.6)$ & $36(50.0)$ & \\
\hline \multicolumn{4}{|c|}{ Metabolic syndrome } \\
\hline No & $22(75.9)$ & $16(22.2)$ & \multirow[t]{2}{*}{$<0.001^{*}$} \\
\hline Yes & $7(24.1)$ & $56(77.8)$ & \\
\hline \multicolumn{4}{|c|}{ ACE inhibitor use } \\
\hline No & $17(58.6)$ & $47(65.3)$ & \multirow[t]{2}{*}{0.530} \\
\hline Yes & $12(41.4)$ & $25(34.7)$ & \\
\hline \multicolumn{4}{|l|}{ ARB use } \\
\hline No & $14(48.3)$ & $31(43.1)$ & \multirow[t]{2}{*}{0.633} \\
\hline Yes & $15(51.7)$ & $41(56.9)$ & \\
\hline \multicolumn{4}{|l|}{$\beta$-blocker use } \\
\hline No & $12(41.4)$ & $33(45.8)$ & \multirow[t]{2}{*}{0.684} \\
\hline Yes & $17(58.6)$ & $39(54.2)$ & \\
\hline \multicolumn{4}{|l|}{ CCB use } \\
\hline No & $15(51.7)$ & $42(58.3)$ & \multirow[t]{2}{*}{0.544} \\
\hline Yes & $14(48.3)$ & $30(41.7)$ & \\
\hline \multicolumn{4}{|l|}{ Thiazide use } \\
\hline No & $23(78.3)$ & $57(79.2)$ & \multirow[t]{2}{*}{0.987} \\
\hline Yes & $6(20.7)$ & $5(20.8)$ & \\
\hline \multicolumn{4}{|l|}{ Statin } \\
\hline No & $12(41.4)$ & $31(43.1)$ & \multirow[t]{2}{*}{0.877} \\
\hline Yes & 17 (58.6) & 35 (56.9) & \\
\hline \multicolumn{4}{|l|}{ Fibrate } \\
\hline No & $23(79.3)$ & 64 (88.9) & \multirow[t]{2}{*}{0.208} \\
\hline Yes & $6(20.7)$ & $5(11.3)$ & \\
\hline
\end{tabular}

Data are expressed as number of patients and analysis was performed by using the Chi square test

$A R B$ angiotensin-receptor blocker, $A C E$ angiotensin-converting enzyme, $C C B$ calcium-channel blocker

* $P<0.05$ was considered statistically significant

gender, age, and BMI as well as glucose, TG, HDL-C levels, and GFR [4]. Our study also revealed that lower adiponectin, elevated insulin and HOMA-IR values, higher CRP, fasting glucose, TG, and lower HDL-C levels and increased BW, WC, BMI, SBP, and bilateral baPWV values exhibited a statistically significant difference between patients with hypertensive MetS and those without MetS, suggesting that circulating adiponectin concentration has association with insulin resistance and other metabolic risks.

Arterial stiffness, which is associated with low-grade inflammation and vascular element reorganization with unregulated collagen and elastin fiber, can be assessed simply and noninvasively by measuring PWV [24]. Many studies have demonstrated that PWV is a strong independent prognostic factor of $\mathrm{CV}$ disease as well as mortality [7-10]. Epidemiological studies revealed that SBP, TG, and HDL-C are independent predictors of baPWV in 835 young adults and MetS and its components (fasting glucose and BP) had an independent association with the incidence or future progression of arterial stiffness among 1518 community-dwelling Taiwanese adults aged over 40 years [25]. Other studies also stated that age, BMI, BP, prevalence of antihypertensive medication, and renal function were significantly associated with arterial stiffness, and increased baPWV values were noted while these risk factors accumulated [26-28]. In addition, stiffness of large conduit arteries causes elevated SBP and decreased DBP, resulting in increasing pulse pressure and PWV, a vicious circle for 
Table 4 Clinical variables with arterial stiffness in the hypertensive patients with or without metabolic syndrome

\begin{tabular}{|c|c|c|c|c|c|c|}
\hline \multirow[t]{2}{*}{ Variables } & \multicolumn{3}{|c|}{ No metabolic syndrome $(n=38)$} & \multicolumn{3}{|c|}{ Metabolic syndrome $(n=63)$} \\
\hline & $\begin{array}{l}\text { Low AS group } \\
(n=22)\end{array}$ & $\begin{array}{l}\text { High AS group } \\
(n=16)\end{array}$ & $P$ value & Low AS group $(n=7)$ & $\begin{array}{l}\text { High AS group } \\
(n=56)\end{array}$ & $P$ value \\
\hline Age (years) & $63.00 \pm 8.94$ & $70.44 \pm 10.37$ & $0.023^{*}$ & $67.14 \pm 8.65$ & $63.75 \pm 9.30$ & 0.363 \\
\hline Height (cm) & $161.237 \pm 5.95$ & $163.59 \pm 8.73$ & 0.326 & $157.00 \pm 8.72$ & $161.16 \pm 8.98$ & 0.251 \\
\hline Body weight (kg) & $62.36 \pm 11.20$ & $64.69 \pm 10.42$ & 0.520 & $67.43 \pm 5.65$ & $74.36 \pm 13.23$ & 0.178 \\
\hline Waist circumference (cm) & $84.09 \pm 11.04$ & $85.81 \pm 6.88$ & 0.586 & $92.14 \pm 4.18$ & $99.32 \pm 9.70$ & 0.646 \\
\hline Body mass index $\left(\mathrm{kg} / \mathrm{m}^{2}\right)$ & $23.95 \pm 3.77$ & $24.05 \pm 2.44$ & 0.930 & $27.46 \pm 2.67$ & $28.45 \pm 3.34$ & 0.454 \\
\hline Left baPWV (m/s) & $10.64 \pm 2.41$ & $15.43 \pm 0.86$ & $<0.001^{*}$ & $13.13 \pm 0.98$ & $17.22 \pm 2.45$ & $<0.001^{*}$ \\
\hline Right baPWV (m/s) & $10.56 \pm 2.52$ & $15.06 \pm 1.03$ & $<0.001^{*}$ & $13.00 \pm 1.13$ & $17.55 \pm 2.66$ & $<0.001^{*}$ \\
\hline $\begin{array}{l}\text { Systolic blood pressure } \\
(\mathrm{mmHg})\end{array}$ & $122.50 \pm 13.87$ & $136.06 \pm 8.97$ & $0.002^{*}$ & $127.86 \pm 18.14$ & $137.68 \pm 17.39$ & 0.166 \\
\hline $\begin{array}{l}\text { Diastolic blood pressure } \\
(\mathrm{mmHg})\end{array}$ & $69.50 \pm 9.53$ & $76.25 \pm 11.14$ & 0.052 & $73.00 \pm 7.53$ & $76.20 \pm 10.97$ & 0.458 \\
\hline Pulse pressure (mmHg) & $53.00 \pm 12.95$ & $59.81 \pm 11.62$ & 0.104 & $54.86 \pm 17.40$ & $61.48 \pm 15.74$ & 0.303 \\
\hline Total cholesterol (mg/dL) & $178.14 \pm 37.05$ & $175.31 \pm 44.50$ & 0.832 & $181.43 \pm 52.21$ & $171.25 \pm 40.55$ & 0.546 \\
\hline Triglycerides (mg/dL) & $107.00(72.25-128.50)$ & $101.00(76.25-138.00)$ & 0.429 & $65.00(45.00-164.00)$ & $153.00(114.75-220.25)$ & $0.026^{*}$ \\
\hline $\mathrm{HDL}-\mathrm{C}(\mathrm{mg} / \mathrm{dL})$ & $49.00 \pm 12.39$ & $51.56 \pm 16.78$ & 0.591 & $53.86 \pm 9.01$ & $40.55 \pm 10.83$ & $0.003^{*}$ \\
\hline LDL-C (mg/dL) & $107.41 \pm 28.40$ & $102.06 \pm 36.51$ & 0.614 & $104.14 \pm 38.86$ & $100.11 \pm 30.95$ & 0.753 \\
\hline Fasting glucose (mg/dL) & $98.00(92.50-121.25)$ & $95.50(89.00-106.00)$ & 0.636 & $113.00(96.00-124.00)$ & $123.50(104.25-167.00)$ & 0.344 \\
\hline $\begin{array}{l}\text { Blood urea nitrogen (mg/ } \\
\mathrm{dL} \text { ) }\end{array}$ & $16.00 \pm 3.60$ & $16.31 \pm 4.56$ & 0.815 & $19.00 \pm 10.55$ & $18.02 \pm 5.93$ & 0.709 \\
\hline Creatinine $(\mathrm{mg} / \mathrm{dL})$ & $1.01 \pm 0.27$ & $1.19 \pm 0.28$ & 0.059 & $1.17 \pm 0.49$ & $1.15 \pm 0.33$ & 0.890 \\
\hline $\begin{array}{l}\text { Glomerular filtration rate } \\
(\mathrm{mL} / \mathrm{min})\end{array}$ & $79.23 \pm 18.66$ & $64.00 \pm 17.66$ & $0.016^{*}$ & $64.86 \pm 24.69$ & $66.80 \pm 19.61$ & 0.811 \\
\hline Total calcium (mg/dL) & $9.03 \pm 0.31$ & $9.08 \pm 0.48$ & 0.864 & $9.22 \pm 0.46$ & $9.20 \pm 0.35$ & 0.850 \\
\hline Phosphorus (mg/dL) & $3.39 \pm 0.53$ & $3.40 \pm 0.37$ & 0.953 & $3.66 \pm 0.40$ & $3.56 \pm 0.57$ & 0.677 \\
\hline Ca X IP product $\left(\mathrm{mg}^{2} / \mathrm{dL}^{2}\right)$ & $30.64 \pm 5.07$ & $30.81 \pm 4.17$ & 0.912 & $33.77 \pm 4.49$ & $32.81 \pm 5.59$ & 0.663 \\
\hline iPTH (pg/mL) & $49.20(32.78-64.83)$ & $50.35(36.13-74.60)$ & 0.881 & $56.80(37.90-75.90)$ & $43.70(27.00-54.40)$ & 0.389 \\
\hline C-reactive protein $(\mathrm{mg} / \mathrm{dL})$ & $0.16(0.12-0.19)$ & $0.17(0.12-0.20)$ & 0.581 & $0.17(0.12-0.20)$ & $0.25(0.18-0.38)$ & $0.015^{*}$ \\
\hline Insulin (ulU/mL) & $9.68(5.64-19.54)$ & $10.11(6.26-13.67)$ & 0.662 & $9.61(5.05-17.91)$ & $16.75(9.56-29.32)$ & 0.155 \\
\hline HOMA1-IR & $2.56(1.59-5.01)$ & $2.34(1.41-3.80)$ & 0.791 & $3.34(1.53-4.67)$ & $4.77(2.98-9.22)$ & 0.165 \\
\hline HOMA2-IR & $1.27(0.79-2.48)$ & $1.35(0.81-1.81)$ & 0.919 & $1.48(0.71-2.31)$ & $2.17(1.36-3.91)$ & 0.114 \\
\hline Adiponectin $(\mu \mathrm{g} / \mathrm{mL})$ & $38.98(28.29-50.67)$ & $35.29(25.62-44.77)$ & 0.272 & $44.71(24.14-61.15)$ & $25.41(20.66-32.69)$ & $<0.001^{*}$ \\
\hline
\end{tabular}

Values for continuous variables given as mean \pm standard deviation and compared by Student's $t$ test; variables not normally distributed given as medians and interquartile range and compared by Mann-Whitney $\mathrm{U}$ test

AS arterial stiffness, baPWV brachial-ankle pulse wave velocity, HDL-C high-density lipoprotein cholesterol, LDL-C low-density lipoprotein cholesterol, iPTH intact parathyroid hormone, CaXIP product Calcium-phosphorous product HOMA-IR homeostasis model assessment of insulin resistance

${ }^{*} P<0.05$ was considered statistically significant

Table 5 Multivariate logistic regression analysis to determine factors correlated to arterial stiffness

\begin{tabular}{|c|c|c|c|c|c|c|c|c|c|}
\hline \multirow[t]{2}{*}{ Variables } & \multicolumn{3}{|c|}{ Hypertensive patients $(n=101)$} & \multicolumn{3}{|c|}{ Hypertension without MS $(n=38)$} & \multicolumn{3}{|c|}{ Hypertension with MS ( $n=63$ ) } \\
\hline & OR & $95 \% \mathrm{Cl}$ & $P$ value & OR & $95 \% \mathrm{Cl}$ & $P$ value & OR & $95 \% \mathrm{Cl}$ & $P$ value \\
\hline Adiponectin $(\mu \mathrm{g} / \mathrm{mL})$ & 0.932 & $0.881-0.985$ & $0.012^{*}$ & - & - & - & 0.909 & $0.931-0.996$ & $0.040^{*}$ \\
\hline $\mathrm{SBP}(\mathrm{mmHg})$ & 1.059 & $1.008-1.113$ & $0.022^{*}$ & 1.126 & $1.024-1.237$ & $0.014^{*}$ & - & - & - \\
\hline GFR (mL/min) & - & - & - & 0.858 & $0.739-0.996$ & $0.043^{*}$ & - & - & - \\
\hline
\end{tabular}

Multivariate logistic regression analysis of diabetes, age, waist circumference, body weight, body mass index, systolic blood pressure, diastolic blood pressure, pulse pressure, triglyceride, HDL-C, glomerular filtration rate, CRP, insulin, HOMA1-IR, HOMA2-IR, and adiponectin

$O R$ odds ratio, $\mathrm{Cl}$ confidence interval, SBP systolic blood pressure, $H D L$-C high density lipoprotein cholesterol, CRP C-reactive protein, HOMA-IR homeostasis model assessment of insulin resistance

* $P<0.05$ was considered statistically significant 
developing future CV events [29]. Moreover, old age as well as MetS components may accelerate arterial stiffness via activating extracellular matrix metalloproteinases, a major determinant of vascular remodeling and arterial stiffness [30, 31]. Our study also revealed that lower adiponectin levels, accompanied by traditional risk factors, such as lower GFR, elevated insulin and HOMA-IR values, higher CRP and TG and lower HDL-C levels, and increased BW, WC, BMI, SBP, DBP, pulse pressure, and bilateral baPWV values, demonstrated statistically significant differences between patients with hypertensive high arterial stiffness and those with low arterial stiffness. Moreover, patients with high arterial stiffness had significantly higher rate of diabetes and MetS.

Hypoadiponectinemia is involved in the pathophysiology of atherosclerosis. In a study of 445 Chinese participants aged $\geq 40$ years, circulating adiponectin levels were independently associated with baPWV in a communitybased population after adjustment for gender, age, BMI, renal clearance, and number of MetS components [32]. In line with our findings, a report by Kawamoto et al. revealed that lower serum high-molecular-weight adiponectin was significantly associated with elevated mean PWV as well as BMI, SBP, DBP, and GFR after multiple linear regressions analyzes for mean PWV [25]. Besides, circulating high-molecular-weight adiponectin levels also had a dose-dependent association with mean PWV and exerted greater influence than alterations in BP levels over arterial stiffness mechanics [25]. Furthermore, hypoadiponectinemia is a marker of oxidized low-density lipoproteins (oxLDL), inducing extensive endothelial dysfunction [33]. In the presence of inflammatory and oxidative vascular injury, hypoadiponectinemia causes vascular dysfunction and enhances PWV elevation [12].

Subgroup analysis of our data showed that bilateral baPWV values were significantly elevated in the high arterial stiffness group among hypertensive patients with or without MetS. In addition, serum adiponectin, CRP, TG, and HDL-C levels were significantly associated with high arterial stiffness than lower arterial stiffness in MetS patients, but this association was not prominent in the non-MetS group. Furthermore, MetS patients with high arterial stiffness revealed even further increased left and right baPWV values and CRP levels as well as decreased serum adiponectin levels than non-MetS patients with high arterial stiffness, indicating that these metabolic profiles had a more prominent influence on arterial stiffness in the MetS population. Multivariable analysis of the determining factors correlated with arterial stiffness in our study also confirmed that hypoadiponectinemia is a key independent predictor of arterial stiffness among hypertensive patients. When we take MetS into consideration, a lower serum adiponectin level is still a strong risk factor for the development of arterial stiffness. However, adiponectin level was not an independent factor, and only SBP and GFR are crucial independent predictors of arterial stiffness among hypertensive patients without MetS. Our findings are consistent with previous study reported by Chen et al. that baPWV was significantly increased in a south Chinese population with MetS relative to those without MetS [34].

There are some limitations to the current study. Firstly, this was a cross-sectional study and therefore, further longitudinal studies are needed before a cause-effect relationship between serum adiponectin and arterial stiffness can be established in the hypertensive population. Secondly, adiponectin has three different oligomeric forms in the circulation: low-molecular weight trimers, medium-molecular weight hexamers, and highmolecular-weight multimers of 12-18 subunits. Many studies have stated that high-molecular-weight adiponectin is the most active and powerful adiponectin, a strong association between serum total and high-molecular-weight adiponectin was reported [21, 35]. In addition, both circulating total and high-molecular-weight adiponectin levels have been inversely associated with parameters of insulin resistance, endothelial dysfunction, and inflammation, as well as with MetS prevalence or incidence, indicating that the use of total adiponectin concentration is a well-accepted surrogate of MetS and arterial stiffness evaluation [4, 21]. Moreover, the baPWV value is largely dependent on current SBP and careful attention must be paid when evaluates peripheral arterial stiffness by using the baPWV [36]. Therefore peripheral arterial stiffness evaluated by baPWV value may be overestimated in hypertensive patients. Further studies are required to elucidate the relationship between baPWV value and adiponectin levels in hypertensive patients.

\section{Conclusions}

Adiponectin is recognized as a consistent and significant parameter associated with MetS. Taken together, our results indicate that hypoadiponectinemia is positively associated with peripheral AS in hypertensive patients. Further prospective studies are needed to confirm the mechanisms underlying this association.

\section{Additional file}

Additional file 1: Table S1. Correlation between left brachial-ankle pulse wave velocity levels and clinical variables among 101 hemodialysis patients. Table S2. Correlation between right brachial-ankle pulse wave velocity levels and clinical variables among 101 hemodialysis patients.

\section{Abbreviations}

MetS: metabolic syndrome; AS: arterial stiffness; baPWV: brachial-ankle pulse wave velocity; HDL-C: high-density lipoprotein cholesterol; TG: triglycerides; 
DM: diabetes mellitus; CV: cardiovascular; IR: insulin resistance; PWV: pulse wave velocity; BP: blood pressure; SBP: systolic blood pressure; DBP: diastolic blood pressure; CAD: coronary artery disease; BW: body weight; WC: waist circumference; BMI: body mass index; BNU: blood urea nitrogen; Cre: creatinine; TCH: total cholesterol; LDL-C: low-density lipoprotein cholesterol; CRP: C-reactive protein; iPTH: intact parathyroid hormone; MDRD: the modification of diet in renal disease; GFR: glomerular filtration rate; HOMA-IR: homeostasis model assessment of insulin resistance; ACEi: angiotensin-converting enzyme inhibitors; ARB: angiotensin receptor blockers; CCB: calcium channel blockers; PPAR- $\gamma$ : peroxisome proliferator-activated receptor- $\gamma$.

\section{Authors' contributions}

BGH and JHW conceived and designed the experiments. CFY, YCC, and JHW performed the experiments. CJL, MCC and BGH contributed reagents and analyzed the data. MCC and BGH wrote the manuscript. All authors read and approved the final manuscript.

\section{Author details}

${ }^{1}$ Department of Pediatrics, Buddhist Tzu Chi General Hospital, Hualien, Taiwan. ${ }^{2}$ Department of Nursing, Tzu Chi University of Science and Technology, Hualien, Taiwan. ${ }^{3}$ Division of Cardiology, Buddhist Tzu Chi General Hospital, No. 707, Section 3, Chung-Yang Road, Hualien 97002, Taiwan. ${ }^{4}$ School of Medicine, Tzu Chi University, Hualien, Taiwan. ${ }^{5}$ Division of Nephrology, Buddhist Tzu Chi General Hospital, No. 707, Section 3, Chung-Yang Road, Hualien 97002, Taiwan.

\section{Acknowledgements}

This work was supported by grants from Tzu Chi Hospital (TCRD 101-03) in Taiwan.

\section{Competing interests}

The authors declare that they have no competing interests.

\section{Availability of data and materials}

The datasets used and analysed during the current study are available from the corresponding author on reasonable request.

\section{Consent for publication}

All authors have reviewed the final version of the manuscript and approve it for publications.

\section{Ethics approval and consent to participate}

The study was approved by the Protection of Human Subjects Institutional Review Board of Tzu-Chi University and Hospital (IRB-099-97). All the patients signed the consent form for allowing their information to be used for research.

\section{Publisher's Note}

Springer Nature remains neutral with regard to jurisdictional claims in published maps and institutional affiliations.

Received: 20 March 2017 Accepted: 19 June 2017

Published online: 28 June 2017

\section{References}

1. Vlachopoulos C, Xaplanteris P, Aboyans V, et al. The role of vascular biomarkers for primary and secondary prevention. A position paper from the european society of cardiology working group on peripheral circulation: endorsed by the association for research into arterial structure and physiology (artery) society. Atherosclerosis. 2015;241:507-32.

2. Chen MC, Hsu BG, Lee CJ, et al. Hyperleptinaemia positively correlates with cardiometabolic syndrome in hypertensive patients. Int J Clin Exp Pathol. 2016:9:12959-67.

3. Chang LC, Huang KC, Wu YW, et al. The clinical implications of blood adiponectin in cardiometabolic disorders. J Formos Med Assoc. 2009;108:353-66.

4. Lindberg S, Jensen JS, Bjerre M, et al. Low adiponectin levels at baseline and decreasing adiponectin levels over 10 years of follow-up predict risk of the metabolic syndrome. Diabetes Metab. 2017;43:134-9.
5. Yamauchi T, Kadowaki T. Physiological and pathophysiological roles of adiponectin and adiponectin receptors in the integrated regulation of metabolic and cardiovascular diseases. Int J Obes. 2008;32(Suppl 7):S13-8.

6. Hsu BG, Liou HH, Lee CJ, et al. Serum sclerostin as an independent marker of peripheral arterial stiffness in renal transplantation recipients: a crosssectional study. Medicine. 2016;95:e3300.

7. Yamashina A, Tomiyama H, Arai T, et al. Brachial-ankle pulse wave velocity as a marker of atherosclerotic vascular damage and cardiovascular risk. Hypertens Res. 2003;26:615-22.

8. Yokoyama H, Hirasawa K, Aoki T, et al. Brachial-ankle pulse wave velocity measured automatically by oscillometric method is elevated in diabetic patients with incipient nephropathy. Diabet Med. 2003;20:942-5.

9. Wang JM, Su C, Wang Y, et al. Elevated circulating endothelial microparticles and brachial-ankle pulse wave velocity in well-controlled hypertensive patients. J Hum Hypertens. 2009;23:307-15.

10. Vlachopoulos C, Aznaouridis K, Terentes-Printzios D, et al. Prediction of cardiovascular events and all-cause mortality with brachial-ankle elasticity index: a systematic review and meta-analysis. Hypertension. 2012;60:556-62.

11. Mahmud A, Feely J. Adiponectin and arterial stiffness. Am J Hypertens. 2005:18:1543-8.

12. Tsioufis C, Dimitriadis K, Selima M, et al. Low-grade inflammation and hypoadiponectinaemia have an additive detrimental effect on aortic stiffness in essential hypertensive patients. Eur Heart J. 2007;28:1 162-9.

13. Ho GJ, Lee MC, Lee CJ, et al. Hypoadiponectinemia correlates with arterial stiffness in kidney transplantation patients. Clin Exp Nephrol. 2015;19:534-41.

14. Hsu BG, Shih MH, Chen YC, et al. High Serum osteoprotegerin is associated with arterial stiffness in kidney transplant patients. Tohoku J Exp Med. 2015;236:247-53.

15. Alberti KG, Zimmet P, Shaw J. Metabolic syndrome-a new world-wide definition. a consensus statement from the international diabetes federation. Diabet Med. 2006;23:469-80.

16. Alberti KG, Zimmet PZ. Definition, diagnosis and classification of diabetes mellitus and its complications. Part 1: diagnosis and classification of diabetes mellitus provisional report of a WHO consultation. Diabet Med. 1998;15:539-53.

17. Wang JH, Lee CJ, Hsieh JC, et al. Inverse association of long-acting natriuretic peptide with metabolic syndrome in congestive heart failure patients. Diabetol Metab Syndr. 2013;5:19.

18. Wallace TM, Levy JC, Matthews DR. Use and abuse of HOMA modeling. Diabetes Care. 2004;27:1487-95.

19. Cantley J. The control of insulin secretion by adipokines: current evidence for adipocyte-beta cell endocrine signaling in metabolic homeostasis. Mamm Genome. 2014;25:442-54.

20. Ziemke F, Mantzoros CS. Adiponectin in insulin resistance: lessons from translational research. Am J Clin Nutr. 2010;91:258s-61s.

21. Nakashima R, Yamane K, Kamei N, et al. Low serum levels of total and highmolecular-weight adiponectin predict the development of metabolic syndrome in Japanese-Americans. J Endocrinol Invest. 2011;34:615-9.

22. Saisho $Y$, Hirose H, Roberts $R$, et al. C-reactive protein, high-molecularweight adiponectin and development of metabolic syndrome in the japanese general population: a longitudinal cohort study. PLoS ONE. 2013;8:e73430.

23. Kim JY, Ahn SV, Yoon JH, et al. Prospective study of serum adiponectin and incident metabolic syndrome: the arirang study. Diabetes Care. 2013;36:1547-53.

24. Kawamoto R, Tabara Y, Kohara K, et al. Serum high molecular weight adiponectin correlates with arterial stiffness in community-dwelling persons. Endocr Res. 2011;36:53-63.

25. Li Cl, Kardia SL, Liu CS, et al. Metabolic syndrome is associated with change in subclinical arterial stiffness: a community-based taichung community health study. BMC Public Health. 2011;11:808.

26. Li B, Gao H, Li X, et al. Correlation between brachial-ankle pulse wave velocity and arterial compliance and cardiovascular risk factors in elderly patients with arteriosclerosis. Hypertens Res. 2006;29:309-14.

27. Kawamoto R, Kohara K, Tabara Y, et al. An association between decreased estimated glomerular filtration rate and arterial stiffness. Intern Med. 2008:47:593-8.

28. Miyai N, Arita M, Miyashita K, et al. The influence of obesity and metabolic risk variables on brachial-ankle pulse wave velocity in healthy adolescents. J Hum Hypertens. 2009;23:444-50. 
29. Harvey A, Montezano AC, Touyz RM. Vascular biology of aging-implications in hypertension. J Mol Cell Cardiol. 2015;83:1 12-21.

30. Tsai JP, Hsu BG, Lee CJ, et al. Serum leptin is a predictor for central arterial stiffness in hypertensive patients. Nephrology. 2017. doi:10.1111/ nep.12859.

31. Tan J, Hua Q, Xing X, et al. Impact of the metalloproteinase-9/tissue inhibitor of metalloproteinase-1 system on large arterial stiffness in patients with essential hypertension. Hypertens Res. 2007;30:959-63.

32. Sung SH, Chuang SY, Sheu WH, et al. Relation of adiponectin and highsensitivity c-reactive protein to pulse-wave velocity and $n$-terminal pro-b-type natriuretic peptide in the general population. Am J Cardiol. 2009;103:1411-6.
33. Plant S, Shand B, Elder P, et al. Adiponectin attenuates endothelial dysfunction induced by oxidised low-density lipoproteins. Diab Vasc Dis Res. 2008;: $: 102-8$

34. Chen L, Zhu W, Mai L, et al. The association of metabolic syndrome and its components with brachial-ankle pulse wave velocity in South China. Atherosclerosis. 2015;240:345-50.

35. Saito I, Yamagishi K, Chei CL, et al. Total and high molecular weight adiponectin levels and risk of cardiovascular disease in individuals with high blood glucose levels. Atherosclerosis. 2013;229:222-7.

36. Park HJ, Rho TH, Park CS, et al. The relationship between the acute changes of the systolic blood pressure and the brachial-ankle pulse wave velocity. Korean J Intern Med. 2007;22:147-51.

\section{Submit your next manuscript to BioMed Central and we will help you at every step:}

- We accept pre-submission inquiries

- Our selector tool helps you to find the most relevant journal

- We provide round the clock customer support

- Convenient online submission

- Thorough peer review

- Inclusion in PubMed and all major indexing services

- Maximum visibility for your research

Submit your manuscript at www.biomedcentral com/submit 